\title{
Técnica y resultados con reconstrucción pancreato-gástrica ducto-mucosa en pancreatoduodenectomía*
}

\author{
Drs. HÉCTOR LOSADA M. ${ }^{1,2,3}$, CÉSAR MUÑOZ C. ${ }^{1,2}$, \\ JUAN CONTRERAS R. ${ }^{1}$, LUIS BURGOS S.J. ${ }^{1,2,3}$, JORGE SILVA A. ${ }^{1,23}$ \\ 1 Departamento de Cirugía y Traumatología. Universidad de La Frontera. \\ 2 Cirugía hepatopancreática y biliar. Servicio de Cirugía. Hospital Hernán Henríquez Aravena. \\ 3 Servicio de Cirugía. Clínica Alemana. \\ Temuco, Chile.
}

\begin{abstract}
Duct-to-mucosa pancreaticogastrostomy after pancreatoduodenectomy. Experience in 37 patients
\end{abstract}

Background: Pancreatic reconstruction in pancreatoduodenectomy (PD) has many technical options. Evidence shows no difference in pancreatic fistula rate or mortality between pancretogastrostomy and pancreaticojejunostomy reconstruction. Aim: To report the results of the technique used by our team to perform ductto-mucosa pancreaticogastrostomy (DMPG) in PD. Material and Methods: Follow up of 37 patients aged $53 \pm 12$ years ( $59 \%$ women), subjected to pancreatoduodenectomy, using DMPG. Perioperative complications were reported using the Dindo-Clavien classification. Results: All patients had a pancreatic cancer. The tumor was located in the head or ampulla of Vater in $38 \%$ of patients. The most common histological type was adenocarcinoma in 33 patients (89\%). Seventy three percent of patients did not have regional lymph node involvement (N0). Two patients died (5\%). Postoperative complications were registered in 35\% of patients. Two patients developed pancreatic fistulas, that were type A and B I one patient each, according to the classification of the International Study Group on Pancreatic Fistula. Conclusions: The morbidity and mortality associated with DMPG in PD in the reported cohort are comparable to those reported by other local studies.

Key words: Pancreaticoduodenectomy, pancreatic cancer, pancreatogastrostomy.

\section{Resumen}

Introducción: Para la reconstrucción pancreática en pancreatoduodenectomía (PD) existen diversas técnicas; la evidencia científica no demuestra diferencia en el porcentaje de fístulas pancreáticas ni morbimortalidad entre la reconstrucción con pancreatogastrostomía y pancreatoyeyunostomía. Nuestro objetivo es describir la técnica de pancreato-gastro anastomosis ducto-mucosa (PGADM) y los resultados en términos de morbimortalidad de esta técnica utilizada para la reconstrucción pancreática en PD. Material y Método: Estudio de serie de casos con seguimiento. Se incluyeron pacientes mayores de 15 años que fueron sometidos a PD

*Recibido el 12 de mayo de 2011 y aceptado para publicación el 17 de enero de 2012.

Correspondencia: Dr. Hector Losada M. Casilla 54-D. Temuco, Chile. Fax: (56) 45- 325761. hflosada@ufro.cl. 
y en los cuales se realizó reconstrucción pancreática con PGADM por el equipo de cirugía hepatopancreática y biliar del Hospital Regional de Temuco desde 1996 hasta 2010. Se reportó morbilidad perioperatoria según la clasificación de Dindo-Clavien. Se aplica estadística descriptiva. Resultados: La cohorte está constituida por 37 pacientes, la edad promedio fue $53 \pm 12$ años y el 59\% género masculino. Todos los pacientes tienen confirmación histopatológica de neoplasia, siendo los orígenes más frecuentes la cabeza del páncreas y ampolla de Vater con un 38\%. El tipo histológico más frecuente fue el adenocarcinoma en 33 pacientes (89\%). El 73\% de los pacientes no tenía compromiso de ganglios linfáticos regionales (N0). La morbilidad peri operatoria fue de 35\%. Dos pacientes presentaron fístulas pancreáticas (5,4\%), uno tipo A y otra tipo B según la clasificación de la ISGPF. La mortalidad perioperatoria es de 2 pacientes (5\%). Conclusiones: La morbi-mortalidad asociada a PD con reconstrucción pancreática con PGADM es comparable a la reportada por series nacionales.

Palabras clave: Pancreatoduodenectomía (MeSH), neoplasia pancreática (MeSH), morbilidad (MeSH).

\section{Introducción}

La pancreatoduodenectomía (PD) es una de las técnicas más complejas de la cirugía hepatobiliopancreática. La mortalidad en los últimos años se ha acercado a cifras por debajo al 5\% ${ }^{1-4}$. La morbilidad continúa alrededor del 35\%, y está asociada principalmente a la filtración de la anastomosis pancreática. Desde el año 2000 existen 2 reportes chilenos que muestran mortalidad de 5,2\% y 4\%; con morbilidad de $48 \%$ y $57,5 \%$.

Para la reconstrucción pancreática posterior a una PD existen diversas técnicas; la evidencia científica no muestra diferencias en el porcentaje de fístulas pancreáticas ni morbimortalidad entre la reconstrucción con pancretogastrostomía y pancreatoyeyunostomía ${ }^{7-9}$.

Nuestro objetivo es describir la técnica de pancreato-gastro anastomosis ducto-mucosa (PGADM) y los resultados en términos de morbimortalidad de esta técnica utilizada por nuestro equipo para la reconstrucción pancreática en PD.

\section{Material y Método}

Diseño de estudio: Estudio de serie de casos con seguimiento.

Población: Se incluyeron sujetos mayores de 15 años que fueron llevados a pancreatoduodenectomía con reconstrucción con PGADM por el equipo de cirugía hepatopancreática y biliar del Hospital Regional de Temuco desde 1996 hasta 2010.

\section{Definiciones:}

- Morbilidad: Se definió morbilidad perioperatoria según la clasificación de Dindo-Clavien ${ }^{10}$.

- Fístula pancreática: Se definió y clasificó fístula pancreática de acuerdo a la clasificación del International Study Group of Pancreatic Fistulae $(\mathrm{ISGPF})^{11}$.
Técnica quirúrgica: Un aspecto importante del cuidado perioperatorio incluye el uso de catéter peridural para analgesia post-operatoria. En nuestro hospital se realizaba PD en 2 equipos: un equipo realizaba la fase resectiva y otro la fase reconstructiva, con la disminución del tiempo quirúrgico, actualmente se intenta que un cirujano realice ambas fases.

La fase resectiva se inicia con una maniobra de Kocher amplia y la apertura de la transcavidad de los epiplones para abordar la vena porta (VP) a nivel del cuello pancreático. Una vez que se ha realizado el túnel retropancreático se inicia la resección, teniendo en cuenta que puede haber compromiso lateral de la VP. Realizamos control inicial de la arteria hepática común y gastroduodenal sin seccionarlas, para luego realizar la disección de la vía biliar y la sección pancreática. La realización de preservación pilórica queda a criterio de cada cirujano.

La fase reconstructiva se inicia una vez revisada la hemostasia, realizando en primer tiempo la reconstrucción PGADM. En los últimos casos se ha utilizado magnificación de 2,5 x (Figura 1). No existe un diámetro predeterminado del conducto pancreático, ni contextura pancreática para realizar este tipo de reconstrucción. La magnificación de 2,5 x ayuda a realizar la anastomosis en conductos pancreáticos finos. Si el cirujano considera que a pesar de la magnificación o por alguna circunstancia anatómica es imposible realizar este tipo de reconstrucción, se realiza la reconstrucción pancreática con técnica invaginante bien sea al estómago o al yeyuno. En los últimos 5 años esta situación sólo ha sucedido en 2 cirugías.

Inicialmente se evalúa la capacidad del muñón pancreático para realizar la anastomosis sin tensión en la cara posterior del estómago. En caso de tensión se procede a disecar y liberar la parte proximal del muñón pancreático de la VP y la vena esplénica. Esta maniobra de liberación del muñón pancreático permite tener una mejor exposición para realizar 


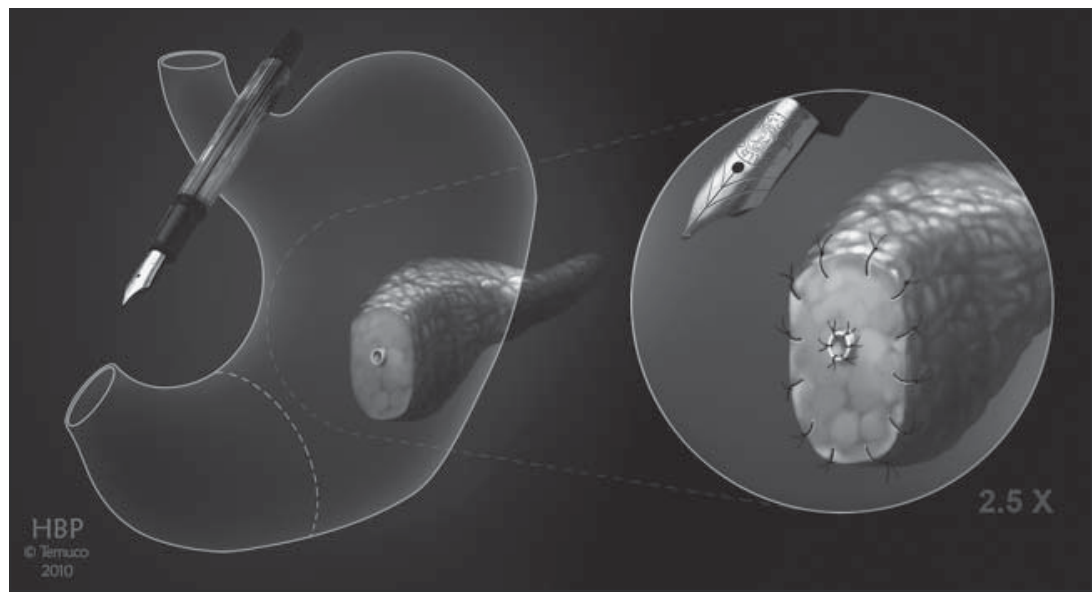

Figura 1. Reconstrucción pancreato-gástrica ductomucosa en pancreatoduodenectomía. El círculo muestra el efecto real de magnificación del campo operatorio con $2.5 \mathrm{X}$.

la anastomosis. La anastomosis se inicia con un primer plano posterior de sutura separada serosapancreático con seda 2-0 ó 3-0 dependiendo de la consistencia del muñón pancreático.

Posteriormente, se realiza una pequeña apertura en la pared gástrica posterior con la punta de aguja del electrobisturí. Se realiza la pared posterior de la anastomosis ducto-mucosa con sutura separada de prolene 6-0 o PDS 6-0. Normalmente se utilizan 3 a 4 puntos en cada pared. Una vez terminada la pared posterior se instala un tutor trans-anastomótico 4 ó 5 French que se deja libre en la cavidad gástrica sin exteriorizarlo, el cual no se fija a ninguna estructura vecina. Posteriormente, se completa la pared anterior de la misma manera. La anastomosis se termina completando el plano restante seroso-pancreático (Figura 2).

Completando la reconstrucción con gastroyeyunanastomosis término-lateral y hepatoyeyunoanstomosis término-lateral, dejando un drenaje tipo Jackson-Pratt en relación a la PGADM.

Plan de análisis: Se utilizó estadística descriptiva, con cálculo de medidas de tendencia central, dispersión y tendencia extrema. Se utilizó en programa STATA ${ }^{\circledR} 9.0$.

\section{Resultados}

La cohorte está constituida por 37 pacientes, con edad promedio de $53 \pm 12$ años (25-74). El 59\% es de género masculino. El 80\% presentó ictericia clínica al momento de la consulta y el síntoma más frecuente de consulta fue el dolor abdominal o dorsal en $70 \%$.

En 35 pacientes se realizó pancreatoduodenecto- mía sin preservación del píloro. En esta serie no se realizó ninguna técnica de resección, ni reconstrucción vascular.

El total de la cohorte tiene confirmación histopatológica de neoplasia, siendo los orígenes más frecuentes la cabeza del páncreas y ampolla de Vater con un 38\% (Tabla 1). El tipo histológico más frecuente fue el adenocarcinoma en 33 pacientes (89\%), teniendo 1 caso de carcinoma indiferenciado, metástasis de melanoma, tumor osteoclástico y un tumor de células gigantes.

Al realizar la estadificación según TNM para cada tipo de tumor encontramos que 24 pacientes fueron T3 (89\%), siete T1 (19\%) y seis T2 (16\%).

El 73\% de la cohorte no tenía compromiso de ganglios linfáticos regionales (N0).

Del total de pacientes, 13 presentaron morbilidad operatoria (35\%), el detalle de estas se presenta en la Tabla 2.

Dos pacientes presentaron fístulas pancreáticas, uno tipo A y otra tipo B según la clasificación de la ISGPF.

Según la Clasificación de Dindo-Clavien, las complicaciones más frecuentes fueron complicación Tipo I y IVA con un 23\% cada una (Tabla 3).

La mortalidad perioperatoria de la cohorte es de 2 pacientes (5\%). Las causas de mortalidad fueron disfunción orgánica múltiple asociada a hemorragia en el segundo día post-quirúrgico y sepsis abdominal sin evidenciar filtración de la anastomosis pancreática. Desde el año 2000 no se presenta mortalidad asociada a PD.

Con un seguimiento promedio de $25 \pm 7,2$ meses, con mediana de 13 meses, la sobrevida global de la serie a 5 años es de 35\%; con intervalo de confianza del 95\% [IC 95] (15\%; 50\%) (Figura 3). 

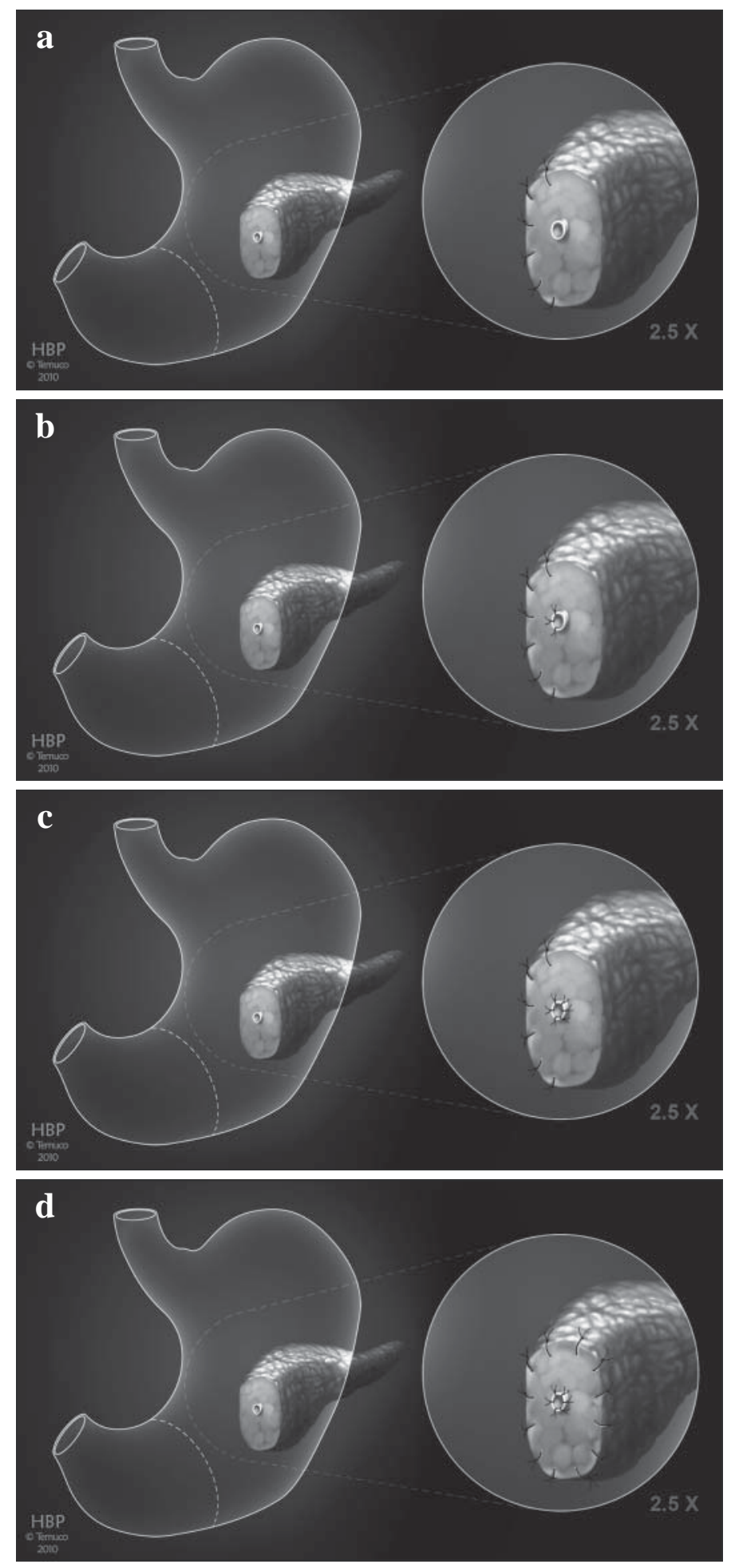

Figura 2. Planos de la anastomosis pancreato-gástrica ducto mucosa: primer plano posterior seroso gástrico-pancreático (a); segundo plano posterior ducto-mucoso (b); tercer plano anterior ducto-mucoso (c); cuarto plano anterior seroso gástrico-pancreático.
Tabla 1.

\begin{tabular}{|lrr|}
\hline $\begin{array}{l}\text { Ubicación de los } \\
\text { tumores }\end{array}$ & $\mathbf{n}$ & $\mathbf{\%}$ \\
\hline Cabeza de páncreas & 14 & 38 \\
\hline Ampolla de Vater & 14 & 38 \\
\hline Colédoco distal & 7 & 19 \\
\hline Duodeno & 2 & 5 \\
\hline Total & 37 & 100 \\
\hline
\end{tabular}

Tabla 2.

\begin{tabular}{|lcc|}
\hline Morbilidad operatoria & $\mathbf{n}$ & $\mathbf{\%}$ \\
$\begin{array}{l}\text { Infección del sitio } \\
\text { operatorio }\end{array}$ & 3 & 23 \\
Colección intraabdominal & 2 & 15 \\
\hline Hemoperitoneo & 1 & 8 \\
\hline Fístula pancreática & 2 & 15 \\
\hline Hemorragia digestiva & 1 & 8 \\
\hline Perforación colónica & 1 & 8 \\
\hline Fístula biliar & 1 & 8 \\
\hline Neumonía & 2 & 15 \\
\hline Total & 13 & 100 \\
\hline
\end{tabular}

Tabla 3.

\begin{tabular}{|lcc|}
\hline $\begin{array}{l}\text { Clasificación de } \\
\text { morbilidad operatoria }\end{array}$ & $\mathbf{n}$ & $\mathbf{\%}$ \\
\hline Tipo I & 3 & 23 \\
\hline Tipo II & 1 & 8 \\
\hline Tipo IIIA & 2 & 15 \\
\hline Tipo IIIB & 2 & 15 \\
\hline Tipo IVA & 3 & 23 \\
\hline Tipo IVB & - & - \\
\hline Tipo V & 2 & 15 \\
\hline Total & 13 & 100 \\
\hline
\end{tabular}




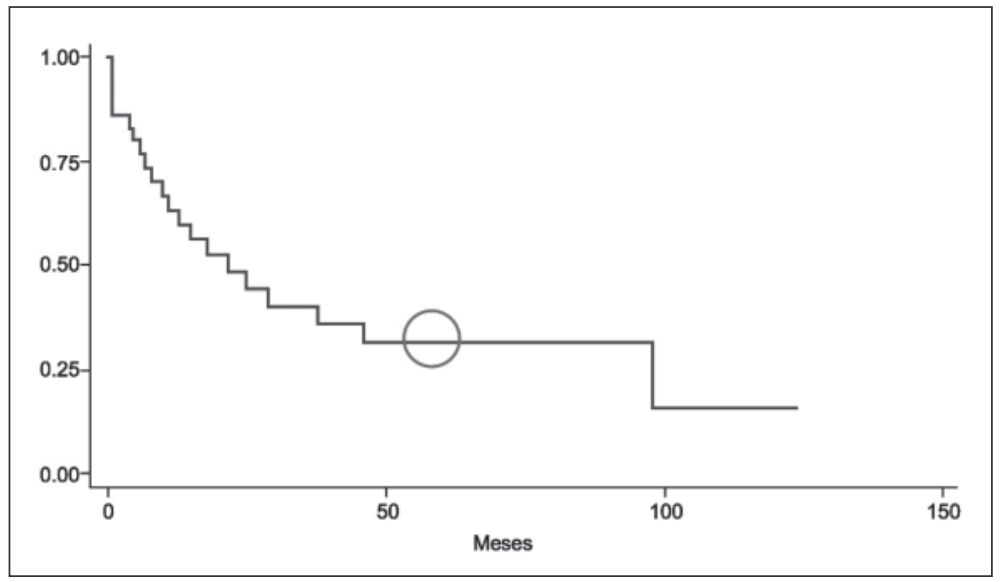

Figura 3. Probabilidad de sobrevida (método de Kaplan-Meier) de la serie a 5 años.

\section{Discusión}

La mayor tasa de morbilidad y mortalidad en PD está relacionada con las complicaciones de la reconstrucción pancreática y existen múltiples opciones para realizarla. En un reporte multicéntrico reciente de 718 PD realizadas en India en el período comprendido 2005 y 2007, se demostró la preferencia de los cirujanos por realizar pancreatoyeyunostomía para la reconstrucción pancreática (68,3\%) por sobre la pancreatogastrostomía, en este reporte no se precisó el tipo de técnica realizada ni el uso de prótesis transanastomótica ${ }^{3}$.

La anastomosis pancreatogástrica para la reconstruccción pancreática se puede realizar ductomucosa o invaginante (dunking).

$\mathrm{Al}$ analizar la evidencia disponible comparando reconstrución pancreatogástrica versus pancreatoyeyuno, podemos decir que no existe mayor beneficio de una por sobre la otra en términos de morbilidad; sin embargo, existen limitaciones en estos análisis ${ }^{8}$. Estudios más recientes, han evidenciado la falta de criterios uniformes para definir fístula pancreática, así como la heterogeneidad en las técnicas empleadas para la reconstrucción ${ }^{12}$. Sin embargo, debemos estar en conocimiento que algunas series de casos recientes que muestran una menor tasa de complicaciones con PGADM ${ }^{13,14}$.

Existen variables clínicas y técnicas que algunas veces no son consideradas al momento de analizar los resultados de la reconstrucción pancreática independiente del tipo de anastomosis y que puede facilitar o dificultar la reconstrucción: consistencia del muñón pancreático, diámetro del conducto pancreático, uso de prótesis trans-anastomótica. Es por este motivo que el International Study Group for Pancreatic Surgery (ISGPS) ha propuesto un nuevo sistema de reporte de las anastomosis pancreáticas, con el fin de estandarizar los reportes y hacer comparables las futuras experiencias ${ }^{3}$.

El ISGPS además ha estandarizado la definición y clasificación de las complicaciones más frecuentes de la PD: fístula pancreática; retardo del vaciamiento gástrico y hemorragia ${ }^{15-18}$. Estas definiciones y clasificaciones, si bien presentan algunas limitaciones, permiten unificar criterios y hacer comparables los resultados entre diferentes grupos ${ }^{12}$.

Aunque sabemos que existen series que no demuestran reducción de la tasa de filtración de la anastomosis pancreática con el uso de prótesis transanastomótica, nuestro grupo ha decidido continuar con este aspecto técnico.

En las diferentes series los factores más importantes asociados a la fístula pancreática son la consistencia del páncreas y el diámetro del conducto pancreático ${ }^{15,19,20}$.

Debemos mencionar que en los últimos 2 años hemos introducido algunos conceptos de recuperación precoz (Fast track), lo cual no se ha asociado a aumento de la morbilidad y ha reducido la estancia hospitalaria. Algunos grupos, como el de la Clínica Universitaria de Navarra, han comunicado resultados comparables a la cirugía tradicional en términos de morbilidad con protocolos para Fast track en $\mathrm{PD}^{21}$.

La sobrevida global a 5 años se presenta a manera descriptiva y no se realizan análisis de sobrevida de cada tipo histológico y origen de tumor por el poco número de pacientes en cada grupo.

Pese a ser un centro de referencia regional, nuestros números nos sitúan como un centro de bajo volumen (37 pacientes en 14 años), donde la cirugía resectiva pancreática la realizan cirujanos con entrenamiento en cirugía hepatobiliopancreática.

El único reporte publicado de un centro regional de alto volumen en PD en Latinoamérica es del 
Instituto Nacional de Ciencias y Nutrición Salvador Zubirán en México, donde se realizaron 122 PD en 6 años; con una mortalidad de $6,5 \%$ y una morbilidad de $41,8 \%{ }^{22}$.

En contraste existen reportes de resultados en centros de bajo volumen que son comparables con resultados de centros de alto volumen; sobre todo en aquellos donde PD fue realizada por cirujanos con entrenamiento ${ }^{23-25}$.

Consideramos que lo importante es mantener un volumen constante de pacientes, la estandarización de la técnica quirúrgica, el registro riguroso de la morbimortalidad y el seguimiento de los pacientes, por el mismo equipo quirúrgico con dedicación y capacitación en cirugía hepatobiliopancreática.

Actualmente, preferimos el manejo no operatorio (percutáneo) de las complicaciones locales de PD, como colecciones asociadas a la anastomosis biliar o pancreática. Si el paciente presenta deterioro clínico a pesar del drenaje adecuado de las colecciones y la terapia antibiótica consideramos la reintervención precoz; teniendo siempre presente que la pancreatectomía total es una opción.

En los casos de filtración de la anastomosis pancreática usamos nutrición parenteral total y sandostatina hasta tener reducción del débito y control de la sepsis. La vía oral se reinicia con base en el débito de la fístula pancreática y el estado del paciente.

Los pacientes con hemoperitoneo en el período post-operatorio temprano o sepsis abdominal de origen no precisado se reintervienen precozmente.

La morbilidad y mortalidad de nuestra serie es similar a las reportadas en series nacionales ${ }^{5,6}$.

\section{Conclusión}

La morbi-mortalidad asociada a PD con reconstrucción pancreática con PGADM es comparable a la reportada por series nacionales.

\section{Referencias}

1. Murakami Y, Uemura K, Hayashidani Y, Sudo T, Hashimoto Y, Nakagawa N, et al. No mortality after 150 consecutive pancreatoduodenctomies with duct-to-mucosa pancreaticogastrostomy. J Surg Oncol. 2008;97:205-9.

2. Schmidt CM, Powell ES, Yiannoutsos CT, Howard TJ, Wiebke EA, Wiesenauer CA, et al. Pancreaticoduodenectomy: a 20-year experience in 516 patients. Arch Surg. 2004;139:718-25; discussion 25-7.

3. Shukla PJ, Barreto SG, Bedi M, Bheerappa N, Chaudhary A, Gandhi M, et al. Peri-operative outcomes for pancreatoduodenectomy in India: a multi-centric study. HPB (Oxford). 2009;11:638-44.
4. Winter JM, Cameron JL, Campbell KA, Arnold MA, Chang DC, Coleman J, et al. 1423 pancreaticoduodenectomies for pancreatic cancer: A single-institution experience. J Gastrointest Surg. 2006;10:1199-210; discussion 210-1.

5. E. W. Pancreatogastroanastomosis en pancreatoduodenectomía. Una alternativa segura de anastomosis al muñon pancreático. Rev Chil Cir. 2002;54:59-64.

6. Benavides C, Burmeister R, García C, Apablaza S, P. R. Resultados inmediatos de la pancreatoyeyunoanastomosis término-lateral con Wirsunostomía tras pancreatoduodenectomía. Rev Chil Cir. 2003;55:567-72.

7. Yeo CJ, Cameron JL, Maher MM, Sauter PK, Zahurak ML, Talamini MA, et al. A prospective randomized trial of pancreaticogastrostomy versus pancreaticojejunostomy after pancreaticoduodenectomy. Ann Surg. 1995; 222:580-8; discussion 8-92.

8. Wente MN, Shrikhande SV, Muller MW, Diener MK, Seiler CM, Friess H, et al. Pancreaticojejunostomy versus pancreaticogastrostomy: systematic review and meta-analysis. Am J Surg. 2007;193:171-83.

9. Yang SH, Dou KF, Sharma N, Song WJ. The methods of reconstruction of pancreatic digestive continuity after pancreaticoduodenectomy: a meta-analysis of randomized controlled trials. World J Surg. 2011;35:2290-7.

10. Clavien PA, Barkun J, de Oliveira ML, Vauthey JN, Dindo D, Schulick RD, et al. The Clavien-Dindo classification of surgical complications: five-year experience. Ann Surg. 2009;250:187-96.

11. Bassi C, Dervenis C, Butturini G, Fingerhut A, Yeo C, Izbicki J, et al. Postoperative pancreatic fistula: an international study group (ISGPF) definition. Surgery. 2005;138:8-13.

12. Reano Paredes G, de Vinatea de Cardenas J, Jiménez Chavarria E. [Pancreaticogastrostomy versus pancreaticojejunostomy after pancreaticoduodenectomy: critical analysis of prospective randomised trials]. Cir Esp. 2011;89:348-55.

13. Kim JH, Yoo BM, Kim WH. Which method should we select for pancreatic anastomosis after pancreaticoduodenectomy? World J Surg. 2009;33:326-32.

14. Wellner U, Makowiec F, Fischer E, Hopt UT, Keck T. Reduced postoperative pancreatic fistula rate after pancreatogastrostomy versus pancreaticojejunostomy. J Gastrointest Surg. 2009;13:745-51.

15. Pratt WB, Callery MP, Vollmer CM, Jr. Risk prediction for development of pancreatic fistula using the ISGPF classification scheme. World J Surg. 2008;32:419-28.

16. Pratt WB, Maithel SK, Vanounou T, Huang ZS, Callery MP, Vollmer CM, Jr. Clinical and economic validation of the International Study Group of Pancreatic Fistula (ISGPF) classification scheme. Ann Surg. 2007; 245:443-51.

17. Wente MN, Veit JA, Bassi C, Dervenis C, Fingerhut A, Gouma DJ, et al. Postpancreatectomy hemorrhage (PPH): an International Study Group of Pancreatic 


\section{TÉCNICA Y RESULTADOS CON RECONSTRUCCIÓN PANCREATO-GÁSTRICA DUCTO-MUCOSA EN ...}

Surgery (ISGPS) definition. Surgery. 2007;142:20-5.

18. Wente MN, Bassi C, Dervenis C, Fingerhut A, Gouma DJ, Izbicki JR, et al. Delayed gastric emptying (DGE) after pancreatic surgery: a suggested definition by the International Study Group of Pancreatic Surgery (ISGPS). Surgery. 2007;142:761-8.

19. Fuks D, Piessen G, Huet E, Tavernier M, Zerbib P, Michot F, et al. Life-threatening postoperative pancreatic fistula (grade C) after pancreaticoduodenectomy: incidence, prognosis, and risk factors. Am J Surg. 2009; 197:702-9.

20. Okabayashi T, Kobayashi M, Nishimori I, Sugimoto T, Onishi S, Hanazaki K. Risk factors, predictors and prevention of pancreatic fistula formation after pancreatoduodenectomy. J Hepatobiliary Pancreat Surg. 2007;14:557-63.

21. Morera-Ocon FJ. [Fast-track experience in cephalic duodenopancreatectomy]. Cir Esp.2011;89:333; author reply 4-5.

22. Chan C, Franssen B, Rubio A, Uscanga L. Pancreaticoduodenectomy in a Latin American country: the transition to a high-volume center. J Gastrointest Surg. 2008;12: 527-33.

23. Schwartz GS, Swan RZ, Ruangvoravat L, Attiyeh FF. Morbidity and mortality after hepatic and pancreatic resections: results from one surgeon at a low-volume urban hospital over thirty years. Am J Surg. 2011; 201:438-44

24. Nienhuijs SW, Rutten HJ, Luiten EJ, van Driel OJ, Reemst PH, Lemmens VE, et al. Reduction of in-hospital mortality following regionalisation of pancreatic surgery in the south-east of the Netherlands. Eur J Surg Oncol. 2010; 36: 652-6.

25. Cocieru A, PF. S. HPB surgery can be safely performed in a community teaching hospital. J Gastrointest Surg. 2010;14:1853-7. 